\title{
Article \\ The Effect of Mycorrhizal Fungi and Organic Fertilizers on Quantitative and Qualitative Traits of Two Important Satureja Species
}

\author{
Asma Bastami ${ }^{1}$, Reza Amirnia ${ }^{1, *}$, R. Z. Sayyed ${ }^{2}\left(\mathbb{D}\right.$ and Hesham A. El Enshasy ${ }^{3,4}$ \\ 1 Department of Plant Production and Genetics, Faculty of Agriculture, Urmia University, \\ Urmia 5756151818, Iran; asmabastami@yahoo.com \\ 2 Department of Microbiology, PSGVP Mandal's Arts, Science and Commerce College, \\ Shahada Maharashtra 425409, India; sayyedrz@gmail.com \\ 3 Institute of Bioproduct Development (IBD), Universiti Teknologi Malaysia (UTM), \\ Johor Bahru 81310, Malaysia; henshasy@ibd.utm.my \\ 4 City of Scientific Research and Technology Application (SRTA), New Burg Al Arab, Alexandria 21934, Egypt \\ * Correspondence: r.amirnia@urmia.ac.ir; Tel.: +98-9144026024
}

\section{check for} updates

Citation: Bastami, A.; Amirnia, R.; Sayyed, R.Z.; Enshasy, H.A.E. The Effect of Mycorrhizal Fungi and Organic Fertilizers on Quantitative and Qualitative Traits of Two Important Satureja Species. Agronomy 2021, 11, 1285. https://doi.org/ 10.3390/agronomy11071285

Academic Editors: Elisa Gamalero, Simone Cantamessa, Patrizia Cesaro and Giorgia Novello

Received: 26 May 2021

Accepted: 21 June 2021

Published: 24 June 2021

Publisher's Note: MDPI stays neutral with regard to jurisdictional claims in published maps and institutional affiliations.

Copyright: (c) 2021 by the authors. Licensee MDPI, Basel, Switzerland. This article is an open access article distributed under the terms and conditions of the Creative Commons Attribution (CC BY) license (https:/ / creativecommons.org/licenses/by/ $4.0 /)$.
Abstract: The quantitative yield and essential oil percentage and composition of two important savory species in response to various fertilizers were explored in a field experiment as a factorial study based on a randomized complete block design with three replications in north Lorestan, Iran, in 2017-2019. The first factor was assigned to three mycorrhizal fungi (Funneliformis mosseae, Rhizophagus irregularis, and Glomus fasciculatum), phosphate biofertilizer (Baravar-2), fish manure $(800 \mathrm{~kg} / \mathrm{ha})$, cattle manure (20 t/ha), vermicompost (5 t/ha), and a control (no fertilization); the second factor was assigned to two savory species, including Satureja khuzestanica and S. rechingeri. The results of the combined analysis of variance for the second and third years showed that the simple effects of fertilizers and species were significant on all recorded traits, except for some constituents of the essential oil. Among the mycorrhizal fungi, R. irregularis and S. khuzestanica outperformed $S$. rechingeri in all traits, except for essential oil content and yield. The interaction between year and species was significant for all traits. The essential oil content of S. rechingeri in the third year (5.1\%) was $18 \%$ higher than that of $S$. rechingeri in the second year $(4.3 \%)$ and $41 \%$ higher than that of $S$. khuzestanica in the third year (3.6\%). According to the results, the foliar application of vermin compost at a rate of $5 \mathrm{t} /$ ha can contribute to the sustainable production of both savory species, improving their growth and essential oil yield.

Keywords: dry weight; essential oil; fish manure; phosphate biofertilizer; vermicompost

\section{Introduction}

Satureja khuzestanica and S. rechingeri from the family Lamiaceae are perennial and aromatic and are two of the invaluable plant species that exclusively belong to the flora of Iran. They grow well in gravelly soils in the arid and sunny regions of southwestern Iran. These two plant species are highly similar to one another [1-3]. They were first recognized as new species in Iranian flora in 1994. Their main habitats include central Zagros, southern Lorestan, eastern Ilam, and northern Lorestan. The genus of savory is spread in the Mediterranean region. It has 15 species in Iran, out of which nine species (S. kallarica, S. sahendica, S. edmondi, S. intermedia, S. rechingeri, S. khuzestanica, S. bachtiarica, S. atropatana, and $S$. isophylla) are exclusively found in Iran; others are also observed in Talesh, Torkamanestan, Turkey, Caucasia, Transcaucasia, and Iraq. The essential oils of the two above-mentioned savory species are rich in carvacrol, and their extracts contain free phenol acids, especially rosmarinic acid, so they have considerable biological activities. Their carvacrol has several biological features, including disinfectant, anti-inflammatory, sedative, antibacterial, antifungal, fermentation, and antioxidant activities [4-7]. 
All over the world, the flowers, leaves, stem, and seeds of most Satureja sp. are traditionally used for various diseases and complications, such as gastrointestinal cramps, nausea, diarrhea, muscle pains, and infectious disease [8]. Because of the aromatic characteristics, the leaves and aerial parts of this genus possess distinctive but pleasant tastes [9].

Presently, research on biofertilizers is expanding due to the high costs of chemical fertilizers and their adverse impacts on the environment. Biofertilizers contain preservatives with a crowded population of one or several microorganisms or their metabolic products and are used to improve soil fertility and supply nutrient requirements of plants in a sustainable agricultural system [10-15]. An example of biofertilizers is mycorrhiza, whose effects on growth and nutrient exchange have been extensively researched. Nooshkam et al. [16] reported that the highest plant dry weight of $S$. khuzestanica was observed in pots containing mycorrhiza. In a study on the effect of mycorrhiza on mint (Mentha arvensis), it was found that inoculation with this fungus increased the fresh and dry weight, plant height, essential oil content, and N, P, and K uptake [17].

Nutrients, including N, P, K, and humic acids, are more abundant in vermicompost than in compost. The advantages of vermicompost over other organic fertilizers are that its structure is well changed and contains much fewer pathogenic microorganisms. The process of humidification is conducted at the maturity stage of vermicompost more extensively, and eventually, the fertilizer produced by this method does not have an unpleasant smell due to its high $\mathrm{C} / \mathrm{N}$ ratio [18]. Heidarpour et al. [19] reported that the application of $2 \mathrm{t} /$ ha vermicompost improved the morpho-physiological parameters of summer savory. In another study, the highest essential oil percentage of $S$. sahendica was obtained from the treatment of $2 t /$ ha vermicompost [20]. Naiji and Souri [21] and Rezvani et al. [22] also reported the positive effects of vermicompost on the growth parameters and essential oil of summer savory.

Phosphate biofertilizer (Baravar-2) contains beneficial phosphate-solubilizing bacteria that acidify the soil and exudate phosphatase enzymes, thereby releasing plant-absorbable phosphate ions from P-containing compounds. The phosphate biofertilizer increases the efficiency of fertilizer uptake and significantly enhances the yield $[10,23,24]$. The application of this fertilizer was assessed to be positive for the growth parameters and essential oil of savory [21].

Fish manure is rich in nutrients, especially $\mathrm{N}$ and $\mathrm{P}$, and performs well in resolving the deficiency of these two elements in orchards and farms [25]. The application of fish manure to the soil as an organic fertilizer has optimal effects on soil physical and chemical features, especially by increasing water availability to plants through increasing soil porosity [18]. The use of emulsified fish waste had positive effects on the growth of radish (Raphanus sativa) [26], Solanum lycopersicum [27], and cowpea Vigna sinensis [28].

Nowadays, the foliar application of nutrients is used to supplement the soil incorporation method for biological and organic compounds with various objectives, including crop improvement, the mitigation of harmful chemicals, and the enhancement of some growth-affecting hormones.

Little research has been conducted on S. khuzestanica and S. rechingeri. Although these two species are native to Iran, since they are rich in medicinally active ingredients, research on improving their cultivation can play a remarkable role in accomplishing the goals of the global pharmaceutical industry. The growing demand of this industry for raw materials and the need for maintaining the optimal productivity of natural plant resources further necessitate investigation into the cultivation and processing of medicinal herbs. Additionally, given the significance of biological and organic fertilizers in sustainable agriculture and environmental conservation and the need for reducing the excessive application of chemical fertilizers, the present study aimed to shed light on the effect of three mycorrhizal species, fish manure, cattle manure, vermicompost, and phosphate biofertilizer on the growth parameters, essential oil content, components, and yield, as well as the leaf N, P, K, and Fe uptake rates of two savory species. 


\section{Materials and Methods}

\subsection{Experiment Set Up, Soil Conditions, and Treatments Application}

The study was conducted in Aleshtar in the north of Lorestan province (Long. $48^{\circ} 15^{\prime} \mathrm{E}$, Lat. $33^{\circ} 49^{\prime} \mathrm{N}$, at the elevation of $1567 \mathrm{~m}$ from sea level, mean monthly temperature of $12.7^{\circ} \mathrm{C}$ ) in three cropping years from 2017 to 2019 as a factorial experiment based on a randomized complete block design with three replications. The first factor was composed of three mycorrhizal fungi (Funneliformis massage, Rhizophagus irregularis, and Glomus fasciculatum), fish manure (800 kg/ha), cattle manure (20 t/ha), vermicompost (5 t/ha), phosphate biofertilizer (Barvar-2) and a control (non-fertilized). Mycorrhiza inoculant was used as a soil fertilizer, and the phosphate biofertilizer was used only as a solution. Fish manure, cattle manure, and vermicompost were applied in both soil and solution form. The second factor was assigned to two savory species, including S. khuzestanica and S. rechingeri. In the winter of 2016, the seedlings of the two species were supplied by the research farm of Khorrman Medicinal Plant Company in Khoramabad, Iran. They were immediately planted in the substrate to produce roots. Then, they were transplanted at the farm in April 2017. Before the treatments were applied, samples were taken from the depth of $0-30 \mathrm{~cm}$ at different farm points and mixed to produce a composite sample to determine the physical and chemical characteristics of the soil in the soil laboratory, Khorrman Company (Table 1).

Table 1. Soil properties at the experimental field.

\begin{tabular}{|c|c|c|c|c|c|c|c|c|c|c|c|}
\hline $\begin{array}{l}\text { Depth } \\
\text { (cm) }\end{array}$ & $\begin{array}{c}\text { Soil } \\
\text { Texture }\end{array}$ & $\begin{array}{l}\text { Clay } \\
(\%)\end{array}$ & $\begin{array}{l}\text { Silt } \\
(\%)\end{array}$ & $\begin{array}{c}\text { Sand } \\
(\%)\end{array}$ & $\mathrm{pH}$ & $\begin{array}{c}\text { EC } \\
(\mathrm{mmho} / \mathrm{cm})\end{array}$ & $\begin{array}{c}\mathrm{N} \\
(\%)\end{array}$ & $\begin{array}{c}\text { Average } P \\
(\mathrm{ppm})\end{array}$ & $\begin{array}{c}\text { Average K } \\
(\mathrm{ppm})\end{array}$ & SP & $\begin{array}{l}\text { OC } \\
(\%)\end{array}$ \\
\hline $0-30$ & Silty-Clay & 26 & 35 & 39 & 6.9 & 1.1 & 0.1 & 10.4 & 250 & 43 & 0.6 \\
\hline
\end{tabular}

The farm was prepared, and furrows were created of $50 \mathrm{~cm}$. Each experimental plot was composed of three sowing rows with a length of $3 \mathrm{~m}$. The plots were separated with an unsown row. The planting arrangement was in the form of $50 \mathrm{~cm} \times 50 \mathrm{~cm}(4$ plants $/ \mathrm{m})$. The blocks were spaced by $1 \mathrm{~m}$. Planting was realized by hand for which $20 \mathrm{~cm}$ holes were first created at the farm, and then, the seedlings were placed in these holes and immediately irrigated to help the rapid establishment of the plants. The initial furrow irrigation was performed every day. However, after the plants were fully established, it was reduced to once a week, according to the climatic conditions. All plots were weeded by hand.

\subsection{Measurement of LAI and Dry Mass Weight}

The vegetative body of the plants was harvested at the flowering phase by hand at the height of $8 \mathrm{~cm}$ from the ground in early October 2018. To measure plant dry mass weight, four plants (from an area of $1 / \mathrm{m}$ ) were sampled after eliminating the marginal lines and $50 \mathrm{~cm}$ from both ends of each row as the margins. The samples were dried in the shade at room temperature $\left(20^{\circ} \mathrm{C}\right)$ for three days, and then they were weighed. The leaf area index (LAI) was measured, using a leaf area meter AM200 (ADC BioScientifc Ltd., Herts Hoddesdon, EN11 0NT, U.K.) in each replication.

\subsection{Essential Oil Isolation, Compound Identification, and Analysis}

To prepare an extract, the fully dried samples were ground. Then, $50 \mathrm{~g}$ of the dry mass sample was used for extraction with a micro-Clevenger by the water-distillation method for three hours in the chemistry laboratory of the Khorrman Company. Next, the essential oil percentage was estimated as per the plant dry mass weight (including leaves and flowering branches). The essential oils were poured into stained glass and dehydrated by using sodium sulfate. Essential oil yield was calculated as the essential oil percentage multiplied by the dry mass weight. For the gas chromatography analysis of the essential oil, a Shimadzu-2014 RTX-5 gas-chromatograph equipped with a capillary column (a height of $30 \mathrm{~m}$, an internal diameter of $0.25 \mathrm{~mm}$, and a thickness of $0.25 \mu \mathrm{m}$ ) was employed. The 
essential oil was analyzed with an Agilent-789I gas-chromatograph coupled with a mass spectrometer equipped with an HP-5MS column with a height of $30 \mathrm{~m}$, an internal diameter of $0.25 \mathrm{~mm}$, and a thickness of $0.25 \mu \mathrm{m}$ [29].

To determine the $\mathrm{N}, \mathrm{P}, \mathrm{K}$, and Fe contents in savory leaves, a $20 \mathrm{~g}$ sample was randomly taken from each plot. After the collected samples were oven-dried at $70{ }^{\circ} \mathrm{C}$ for $48 \mathrm{~h}$, they were powdered with an electric blender and were finally extracted by the digestion method, using sulfuric acid, salicylic acid, hydrogen peroxide, and selenium [30]. The leaf $\mathrm{N}$ content was determined, using the post-digestion titration method with the help of a Tecator Kjltec auto 10 analyzer. The leaf P content was estimated by colorimetry (vanadate-molybdate yellow color), using a Shimadzu-U73100 GBC spectrophotometer. The leaf K content was measured by flame photometry, and the leaf Fe content was determined by a ShimadzuAA6400 atomic absorption device [30]. The mycorrhiza inoculant was a soil mixture containing spores (150 spores per $100 \mathrm{~g}$ dry clay soil), hyphae, and the root fragments of the host plant (clover) and was procured from the commercial laboratory of Turan Biotech, Shahrud, Iran.

The vermicompost used in the study had a plant origin, too. It was procured from Kimia Biotechnology Co., Shahrud, Iran. Table 2 presents the results of its analysis.

Table 2. Physical and chemical properties of fertilizers used in the test.

\begin{tabular}{cccccccc}
\hline Type of Fertilizers & $\begin{array}{c}\text { EC } \\
(\mathbf{m m h o / c m})\end{array}$ & $\mathbf{p H}$ & $\mathbf{O C ~ ( \% )}$ & $\mathbf{N ~ ( \% )}$ & $\mathbf{P}$ & $\mathbf{K}$ & $\mathbf{F e}$ \\
\hline Cattle manure & 4.14 & 9.01 & 22 & 1.87 & 0.14 & $\mathbf{( m g / k g )}$ & \\
\hline Vermicompost & 8.4 & 6.5 & 31 & 1.16 & 0.9 & 1.24 & 891 \\
\hline Fish manure & 5.5 & 6.5 & 5.32 & 0.65 & 0.12 & 0.75 & 500 \\
\hline
\end{tabular}

The phosphate biofertilizer, with a trademark of Barvar-2, was procured from Green Biotech Inc., Tehran, Iran. It contained two bacteria, i.e., strain 15 of Pantoea agglomerans and strain 13 of Pseudomonas putida, with a population of $10^{9}$ colony-forming units (CFU) $\mathrm{cm}^{-3}$. These two bacteria together neutralize soil $\mathrm{pH}$ and release plant-absorbable phosphate, thereby enhancing plant yields. The mycorrhizal inoculant, vermicompost, fish manure, and phosphate biofertilizer (insoluble forms) were placed in the holes during transplanting. It should be noted that since the two studied savory species were perennial, they were sown only in the first year of the experiment. In the second and third experimental years, the same fertilization treatments were applied to the first-year plots, and all operations of the first year (except for transplanting and thinning) were repeated in the second and third years. In order to prepare the foliar application, an organic fertilizer solution was prepared with an organic fertilizer/distilled water ratio of 1:10 so that one kilogram of the organic fertilizer was soaked in $10 \mathrm{~L}$ of distilled water for $48 \mathrm{~h}$. Then, the resulting extract was smoothed with two layers of thin cloth. For the foliar application, first, the field was irrigated in the morning, and then foliar spraying was done in the evening. Thus, the relative humidity of the atmosphere was increased, and the plants had enough time to absorb the solution. The foliar application was performed when the plants were at the 15-leafed stage; it was about 30 days after planting in each growing season.

\subsection{Statistical Analysis}

The first year was regarded as the plant establishment year, and data of the second and third years were subjected to an analysis of variance in the SAS (9.1) software package. The means were also compared by the LSD test at the $p<0.05$ level.

\section{Results}

The results of the combined analysis of variance (ANOVA) revealed that the simple effects of the treatments and species were significant $(p<0.01)$ on plant height, lateral branch number, leaf area index, dry weight, essential oil percentage, essential oil yield, 
and leaf N, P, K, and Fe contents of the savory plants (Table 3). Although the treatments and species significantly influenced carvacrol, their effects were insignificant on other essential oil compounds, such as $\beta$-myrcene, $\alpha$-terpinolene, $\gamma$-terpinene, linalool, thymol acetate, caryophyllene, $\beta$-bisabolene, and para-cymene (Table 4). The interaction of the year and species was significant $(p<0.01)$ for plant height, lateral branch number, dry weight, essential oil percentage, and leaf N, K, and Fe contents (Table 3). Additionally, the interactive effects of the year and treatments were significant on plant height and leaf $\mathrm{P}$ and K contents at the $p<0.01$ level and on leaf Fe content at the $p<0.05$ level (Table 3).

Table 3. Summary of combined analysis of variance on savory studied traits.

\begin{tabular}{|c|c|c|c|c|c|c|c|c|c|c|c|}
\hline S.O.V & df & $\mathbf{H}$ & LB & LAI & DW & EO & EY & $\mathbf{N}$ & $\mathbf{P}$ & $\mathbf{K}$ & $\mathrm{Fe}$ \\
\hline Year & 1 & $95.03 * *$ & $2096.03^{* *}$ & $0.81 * *$ & $0.76^{* *}$ & $13.61^{* *}$ & $1954.64^{* *}$ & $23.26^{* *}$ & $5.98 * *$ & $25.03 * *$ & $166,776.03^{* *}$ \\
\hline Year (rep) & 4 & 49.05 & 283.98 & 0.07 & 3.36 & 0.14 & 57.67 & 2.26 & $0.86^{* *}$ & $0.70 * *$ & 4117.78 \\
\hline Species & 1 & $917.45^{* *}$ & $414.81^{* *}$ & $0.01 * *$ & $18.55^{* *}$ & $45.43 * *$ & $5.30^{\mathrm{ns}}$ & $10.93^{* *}$ & $1.97 * *$ & $9.34 * *$ & $4059.27 * *$ \\
\hline Treatment & 10 & $261^{* *}$ & $351.63 * *$ & $0.26^{* *}$ & $8.08^{* *}$ & $11.45^{* *}$ & $447.17 * *$ & $7.42^{* *}$ & $2.05^{* *}$ & $9.23 * *$ & $67,372.08 * *$ \\
\hline $\begin{array}{l}\text { Species } \times \\
\text { treatment }\end{array}$ & 10 & $3.67^{\mathrm{ns}}$ & $3.03^{\mathrm{ns}}$ & $0.001^{\mathrm{ns}}$ & $0.08^{\mathrm{ns}}$ & $0.14^{\mathrm{ns}}$ & $2.13^{\mathrm{ns}}$ & $0.06^{\mathrm{ns}}$ & $0.05^{\mathrm{ns}}$ & $0.05^{\mathrm{ns}}$ & $386.53^{\mathrm{ns}}$ \\
\hline Year $\times$ species & 1 & $152.75^{* *}$ & $144.27^{* *}$ & $0.003^{\mathrm{ns}}$ & $4.64^{* *}$ & $3.65^{* *}$ & $0.06^{\mathrm{ns}}$ & $2.41^{* *}$ & $0.04^{\mathrm{ns}}$ & $3.12 * *$ & $49,959.27^{* *}$ \\
\hline $\begin{array}{c}\text { Year } \times \\
\text { treatment }\end{array}$ & 10 & $26.01 * *$ & $4.88^{\mathrm{ns}}$ & $0.002^{\mathrm{ns}}$ & $0.25^{\mathrm{ns}}$ & $0.11^{\mathrm{ns}}$ & $8.05^{\mathrm{ns}}$ & $0.07^{\mathrm{ns}}$ & $0.34 * *$ & $0.45^{* *}$ & $1690.06^{*}$ \\
\hline $\begin{array}{c}\text { Year } \times \text { species } \\
\times \text { treatment }\end{array}$ & 10 & $7.57^{\mathrm{ns}}$ & $3.25^{\mathrm{ns}}$ & $0.001^{\mathrm{ns}}$ & $0.01^{\mathrm{ns}}$ & $0.06^{\mathrm{ns}}$ & $2.01^{\mathrm{ns}}$ & $0.08^{\mathrm{ns}}$ & $0.06^{\mathrm{ns}}$ & $0.05^{\mathrm{ns}}$ & $62.83^{\mathrm{ns}}$ \\
\hline Error & 84 & 5.14 & 5.65 & 0.002 & 0.22 & 0.22 & 5.15 & 0.18 & 0.04 & 0.16 & 920.61 \\
\hline $\mathrm{CV}$ & - & 7.29 & 8.2 & 9.10 & 12.0 & 11.26 & 15.2 & 16. 10 & 16.9 & 13.11 & 8.44 \\
\hline
\end{tabular}

** ${ }^{*}$ and ${ }^{\mathrm{ns}}$, significant at $1 \%, 5 \%$, and not significant probability levels, respectively. H, plant height; LB, lateral branches; LAI, leaf area index; DW, dry mass weight; EO, essential oil, EY, Essential oil yield, respectively.

Table 4. Summary of combined analysis of variance of essential oil compositions of savory.

\begin{tabular}{|c|c|c|c|c|c|c|c|c|c|c|}
\hline S.O.V & df & $\begin{array}{c}\beta- \\
\text { Myrcene }\end{array}$ & $\begin{array}{c}\alpha- \\
\text { Terpinolene }\end{array}$ & $\begin{array}{c}\gamma- \\
\text { Terpinene }\end{array}$ & Linalool & Carvacrol & $\begin{array}{l}\text { Thymol } \\
\text { Acetate }\end{array}$ & Caryophyllene & $\begin{array}{c}\beta- \\
\text { Bisabolene }\end{array}$ & $\begin{array}{c}\text { Para- } \\
\text { Cymene }\end{array}$ \\
\hline Year & 1 & $0.02^{\mathrm{ns}}$ & 0.06 ** & $3.6^{* *}$ & $1.38 * *$ & $24.48 * *$ & $0.02^{\mathrm{ns}}$ & $5.77 * *$ & $1.59 * *$ & $8.42 * *$ \\
\hline Year(rep) & 4 & 0.02 & 0.006 & 0.48 & 0.03 & 1.57 & 0.007 & 0.06 & 0.24 & 1.05 \\
\hline Species & 1 & $0.0001^{\mathrm{ns}}$ & $0.0007^{\mathrm{ns}}$ & $0.76^{\mathrm{ns}}$ & $0.01^{\mathrm{ns}}$ & $1673.4^{* *}$ & $0.00003^{\mathrm{ns}}$ & $0.22^{\mathrm{ns}}$ & $0.32^{\mathrm{ns}}$ & $0.006^{\mathrm{ns}}$ \\
\hline Treatment & 10 & $0.007^{\mathrm{ns}}$ & $0.004^{\mathrm{ns}}$ & $0.34^{\mathrm{ns}}$ & $0.05^{\mathrm{ns}}$ & $209.35 * *$ & $0.012^{\mathrm{ns}}$ & $0.4^{\mathrm{ns}}$ & $0.72^{\mathrm{ns}}$ & $0.26^{\mathrm{ns}}$ \\
\hline $\begin{array}{l}\text { Species } \times \\
\text { treatment }\end{array}$ & 10 & $0.006^{\mathrm{ns}}$ & $0.006^{\mathrm{ns}}$ & $0.25^{\mathrm{ns}}$ & $0.17^{\mathrm{ns}}$ & $5.38^{\mathrm{ns}}$ & $0.016^{\mathrm{ns}}$ & $0.36^{\mathrm{ns}}$ & $0.3^{\mathrm{ns}}$ & $0.41^{\mathrm{ns}}$ \\
\hline Year $\times$ species & 1 & $\begin{array}{c}0.00003 \\
\text { ns }\end{array}$ & $0.004^{\mathrm{ns}}$ & $0.007^{\mathrm{ns}}$ & $0.007^{\mathrm{ns}}$ & $3.38^{\mathrm{ns}}$ & $0.010^{\mathrm{ns}}$ & $0.56^{\mathrm{ns}}$ & $0.0006^{\mathrm{ns}}$ & 0.07 \\
\hline $\begin{array}{c}\text { Year } \times \\
\text { treatment }\end{array}$ & 10 & $0.006^{\mathrm{ns}}$ & $0.002^{\mathrm{ns}}$ & $0.099^{\mathrm{ns}}$ & $0.02^{\mathrm{ns}}$ & $0.94^{\mathrm{ns}}$ & $0.012^{\mathrm{ns}}$ & $0.26^{\mathrm{ns}}$ & $0.23^{\mathrm{ns}}$ & $0.43^{\mathrm{ns}}$ \\
\hline $\begin{array}{c}\text { Year } \times \text { species } \\
\times \text { treatment }\end{array}$ & 10 & $0.007^{\mathrm{ns}}$ & $0.006^{\mathrm{ns}}$ & $0.09^{\mathrm{ns}}$ & $0.05^{\mathrm{ns}}$ & $0.40^{\mathrm{ns}}$ & $0.018^{\mathrm{ns}}$ & $0.33^{\mathrm{ns}}$ & $0.18^{\mathrm{ns}}$ & $0.46^{\mathrm{ns}}$ \\
\hline Error & 84 & 0.006 & 0.005 & 0.28 & 0.04 & 5.27 & 0.006 & 0.30 & 0.35 & 0.27 \\
\hline $\mathrm{CV}$ & - & 19.12 & 17.77 & 18.25 & 20.21 & 2.9 & 15.65 & 16.62 & 17.66 & 16.21 \\
\hline
\end{tabular}

** and ${ }^{\mathrm{ns}}$, significant at $1 \%$ and not significant probability levels, respectively.

\subsection{Plant Height}

The comparison of the means revealed that the foliar application of vermicompost was related to the tallest Satureja plants with a height of $39.16 \mathrm{~cm}$. The plants inoculated with $R$. irregularis grew to a height of $36.58 \mathrm{~cm}$, which was significantly different from those inoculated with the other mycorrhizal fungus (Table 5). The application of phosphate biofertilizer $(31.66 \mathrm{~cm})$ did not differ from the inoculation with tha $(32.33 \mathrm{~cm})$, the foliar application of cattle manure, and the soil application of vermicompost significantly. However, all these treatments exhibited a significant difference in plant height from the control plants $(23 \mathrm{~cm})$ (Table 5). The interaction between year and species was significant. The plant height of $S$. khuzestanica in the third year $(35.2 \mathrm{~cm})$ was $3 \%$ higher than that of S. rechingeri in the second year $(28.7 \mathrm{~cm}$ ) (Table 6). Regarding the interaction between year and treatment, it was observed that the savory plants sprayed by vermicompost were $8 \%$ taller in the third year than in the second year ( 40.5 vs. $37.5 \mathrm{~cm}$ ) (Table 7$)$. 
Table 5. Mean comparison of some measured characters of savory.

\begin{tabular}{|c|c|c|c|c|c|c|c|c|c|c|c|}
\hline Treatments & $\begin{array}{c}\mathrm{H} \\
(\mathrm{cm})\end{array}$ & LB & LAI & $\begin{array}{c}\text { DW } \\
\text { (t/ha) }\end{array}$ & $\begin{array}{l}\text { EO } \\
(\%)\end{array}$ & $\begin{array}{c}\text { EY } \\
\text { (kg/ha) }\end{array}$ & $\begin{array}{c}\mathrm{N} \\
(\%)\end{array}$ & $\begin{array}{c}\mathbf{P} \\
(\%)\end{array}$ & $\begin{array}{c}K \\
(\%)\end{array}$ & $\begin{array}{c}\text { Fe } \\
(\mathrm{mg} / \mathrm{kg})\end{array}$ & Carvacrol \\
\hline \multicolumn{12}{|l|}{ Species } \\
\hline Rechingeri & $33.74^{b}$ & $18.12^{b}$ & $0.57^{\mathrm{b}}$ & $2.51^{b}$ & $4.77^{\mathrm{a}}$ & $13.05^{\mathrm{a}}$ & $2.21^{\mathrm{b}}$ & $1.09^{b}$ & $2.6^{b}$ & $353^{b}$ & $75.5^{b}$ \\
\hline Khuzestanica & $28.46^{\mathrm{a}}$ & $21.66^{a}$ & $0.6^{\mathrm{a}}$ & $3.26^{\mathrm{a}}$ & $3.6^{\mathrm{b}}$ & $12.65^{b}$ & $2.78^{\mathrm{a}}$ & $1.33^{\mathrm{a}}$ & $3.13^{\mathrm{a}}$ & $364^{\mathrm{a}}$ & $82.62^{a}$ \\
\hline $\mathrm{LSD}_{5 \%}$ & 0.78 & 0.82 & 0.01 & 0.14 & 0.16 & 0.78 & 0.14 & 0.07 & 0.14 & 10.5 & 0.79 \\
\hline \multicolumn{12}{|l|}{ Year } \\
\hline Year two (2018) & $30.25^{b}$ & $15.9^{b}$ & $0.51^{\mathrm{b}}$ & $2.13^{b}$ & $3.86^{b}$ & $9^{b}$ & $2.08^{b}$ & $1^{b}$ & $2.43^{b}$ & $323.45^{b}$ & $78.63^{b}$ \\
\hline Year three (2019) & $31.36^{\mathrm{a}}$ & $23.87^{\mathrm{a}}$ & $0.67^{\mathrm{a}}$ & $3.65^{\mathrm{a}}$ & $4.5^{\mathrm{a}}$ & $16.7^{\mathrm{a}}$ & $2.92^{\mathrm{a}}$ & $1.42^{\mathrm{a}}$ & $3.24^{\mathrm{a}}$ & $394.76^{\mathrm{a}}$ & $79.49^{a}$ \\
\hline $\mathrm{LSD}_{5 \%}$ & 3.38 & 8.14 & 0.13 & 0.88 & 0.18 & 3.67 & 0.72 & 0.44 & 0.40 & 98.07 & 0.6 \\
\hline \multicolumn{12}{|l|}{ Treatment } \\
\hline Mycorrhiza (F. mosseae) & $33.91^{c}$ & $24.3^{c}$ & $0.71^{b}$ & $3.69^{b c}$ & $5.10^{b c}$ & $18.77^{\mathrm{b}}$ & $3.19^{b c}$ & $1.42^{\mathrm{cd}}$ & $3.62^{b c}$ & $426.5^{b c}$ & $83.16^{\mathrm{b}}$ \\
\hline $\begin{array}{l}\text { Mycorrhiza } \\
\text { (R. irregularis) }\end{array}$ & $36.58^{b}$ & $26.7^{b}$ & $0.76^{\mathrm{a}}$ & $3.83^{\mathrm{ab}}$ & $5.25^{\mathrm{ab}}$ & $20.09^{b}$ & $3.47^{\mathrm{ab}}$ & $1.56^{b c}$ & $3.92^{\mathrm{ab}}$ & $444.83^{\mathrm{ab}}$ & $83.97^{a b}$ \\
\hline $\begin{array}{l}\text { Mycorrhiza } \\
\text { (G. fasciculatum) }\end{array}$ & $32.33^{\text {cde }}$ & $21.75^{\mathrm{d}}$ & $0.67^{b c}$ & $3.36^{c}$ & $4.78^{\mathrm{cd}}$ & $16.10^{\mathrm{c}}$ & $2.86^{\mathrm{cd}}$ & $1.28^{\mathrm{d}}$ & $3.37^{\mathrm{cd}}$ & $407.33^{c}$ & $80.83^{c}$ \\
\hline Fish manure soil & $26.25^{g}$ & $14.66^{\mathrm{h}}$ & $0.43^{\mathrm{f}}$ & $1.98^{\mathrm{f}}$ & $3.23 \mathrm{fg}$ & $6.51^{\mathrm{f}}$ & $1.67^{\mathrm{fg}}$ & $0.75 \mathrm{fg}$ & $1.91^{\mathrm{h}}$ & $277.92^{g}$ & $74.6^{\mathrm{g}}$ \\
\hline Fish manure_solution & $27.58^{\mathrm{fg}}$ & $15.5^{\mathrm{h}}$ & $0.45^{f}$ & $2.16^{\mathrm{f}}$ & $3.19 \mathrm{~g}$ & $7.09^{f}$ & $1.7_{\mathrm{fg}}$ & $0.81^{\mathrm{fg}}$ & $2.05 \mathrm{gh}$ & $292.83 \mathrm{~g}$ & $75.75 \mathrm{~g}$ \\
\hline Cattle manure soil & $28.41^{f}$ & $16.58^{\mathrm{hg}}$ & $0.53^{\mathrm{e}}$ & $2.53^{\mathrm{e}}$ & 3.61 ef & $9.25^{\mathrm{e}}$ & 2 ef & 0.95 ef & $2.37^{\mathrm{fg}}$ & $322.67^{f}$ & $76.41^{\mathrm{fg}}$ \\
\hline Cattle manure solution & $30.66^{\mathrm{e}}$ & $17.66^{\mathrm{fg}}$ & $0.54^{\mathrm{e}}$ & $2.75^{\mathrm{de}}$ & $3.97^{\mathrm{e}}$ & $10.91^{\mathrm{e}}$ & $2.22^{\mathrm{e}}$ & $1.04^{\mathrm{e}}$ & $2.58^{\mathrm{f}}$ & 339 ef & 78.19 ef \\
\hline Vermicompst soil & $32.58^{\mathrm{cd}}$ & 20.91 de & $0.62^{\mathrm{d}}$ & $2.94^{\mathrm{d}}$ & $4.49^{\mathrm{d}}$ & $13.15^{\mathrm{d}}$ & $2.58^{\mathrm{d}}$ & $1.35^{\mathrm{d}}$ & $2.94^{\mathrm{e}}$ & 363.42 de & $80.26^{\mathrm{dc}}$ \\
\hline Vermicompost solution & $39.16^{\mathrm{a}}$ & $29.58^{\mathrm{a}}$ & $0.79^{a}$ & $4.15^{\mathrm{a}}$ & $5.53^{\mathrm{a}}$ & $22.92^{\mathrm{a}}$ & $3.78^{\mathrm{a}}$ & $1.91^{\mathrm{a}}$ & $4.18^{\mathrm{a}}$ & $466.25^{\mathrm{a}}$ & $85.54^{\mathrm{a}}$ \\
\hline Biofertilizer_phosphate & $31.66^{\mathrm{de}}$ & $19.25^{\mathrm{ef}}$ & $0.63^{\mathrm{cd}}$ & $2.89^{\mathrm{d}}$ & $4.43^{\mathrm{d}}$ & $12.1^{\mathrm{d}}$ & $2.63^{\mathrm{d}}$ & $1.63^{a b}$ & $3.12^{\mathrm{ed}}$ & $381.5^{\mathrm{d}}$ & $78.85^{\text {de }}$ \\
\hline $\mathrm{LSD}_{5 \%}$ & 1.84 & 1.93 & 0.04 & 0.33 & 0.38 & 1.84 & 0.34 & 0.17 & 0.3 & 24.63 & 1.86 \\
\hline
\end{tabular}

In each column and for each experimental factor, means with the same letters or means with differences less than LSD do not have statistically significant differences at a 5\% probability level. H, plant height; LB, lateral branches; LAI, leaf area index; DW, dry weight; EO, essential oil, EY, essential oil yield, respectively.

Table 6. Mean comparisons for interaction effects of year $\times$ species for some savory traits.

\begin{tabular}{|c|c|c|c|c|c|c|c|c|c|c|c|c|c|c|}
\hline \multirow{2}{*}{$\begin{array}{l}\text { Traits } \\
\text { Year }\end{array}$} & \multicolumn{2}{|c|}{$\mathbf{H}$} & \multicolumn{2}{|c|}{ LB } & \multicolumn{2}{|c|}{ DW } & \multicolumn{2}{|c|}{ EO } & \multicolumn{2}{|c|}{$\mathbf{N}$} & \multicolumn{2}{|c|}{$\mathbf{K}$} & \multicolumn{2}{|c|}{$\mathrm{Fe}$} \\
\hline & 2 & 3 & 2 & 3 & 2 & 3 & 2 & 3 & 2 & 3 & 2 & 3 & 2 & 3 \\
\hline (Rechingeri) & $28.7^{c}$ & $31.8^{\mathrm{b}}$ & $15.2^{\mathrm{d}}$ & $16.6^{c}$ & $1.9^{\mathrm{d}}$ & $2.3^{c}$ & $4.3^{b}$ & $5.1^{\mathrm{a}}$ & $1.7^{\mathrm{b}}$ & $2.5^{\mathrm{ab}}$ & $2.0^{c}$ & $2.9^{b}$ & $298^{d}$ & $378^{b}$ \\
\hline (Khuzestanica) & $27.6^{c}$ & $35.2^{\mathrm{a}}$ & $20.8^{b}$ & $26.5^{\mathrm{a}}$ & $2.7^{b}$ & $3.7^{\mathrm{a}}$ & $3.4^{c}$ & $3.6^{c}$ & $2.3^{c}$ & $2.7^{\mathrm{a}}$ & $3.1^{b}$ & $3.4^{\mathrm{a}}$ & $348^{\mathrm{c}}$ & $405^{a}$ \\
\hline $\mathrm{LSD}_{5 \%}$ & \multicolumn{2}{|c|}{1.13} & \multicolumn{2}{|c|}{1.16} & \multicolumn{2}{|c|}{0.229} & \multicolumn{2}{|c|}{0.219} & \multicolumn{2}{|c|}{0.254} & \multicolumn{2}{|c|}{0.208} & \multicolumn{2}{|c|}{14.7} \\
\hline
\end{tabular}

Means with the same letters or means with differences less than LSD do not have statistically significant differences at the $5 \%$ level of probability.

Table 7. Mean comparisons for interaction effects of year $\times$ treat for some savory traits.

\begin{tabular}{|c|c|c|c|c|c|c|c|c|}
\hline \multirow{2}{*}{$\begin{array}{c}\text { Traits } \\
\text { Treatments }\end{array}$} & \multicolumn{2}{|c|}{$\mathbf{H}$} & \multicolumn{2}{|c|}{$\mathbf{P}$} & \multicolumn{2}{|c|}{$\mathbf{K}$} & \multicolumn{2}{|c|}{$\mathrm{Fe}$} \\
\hline & 2 & 3 & 2 & 3 & 2 & 3 & 2 & 3 \\
\hline Control & $22.0^{\mathrm{m}}$ & $24.0^{\mathrm{lm}}$ & $0.57 \mathrm{~m}$ & $0.65^{\mathrm{lm}}$ & $1.40^{\mathrm{n}}$ & $1.46^{\mathrm{n}}$ & $219^{1}$ & $237^{\mathrm{kl}}$ \\
\hline Mycorrhiza (F. mosseae) & $30.8^{\text {efg }}$ & $36.5^{b c}$ & $1.07 \mathrm{gh}$ & $1.77 \mathrm{bc}$ & $3.01 \mathrm{fg}$ & $4.25^{\mathrm{bc}}$ & $380^{f}$ & $473^{b c}$ \\
\hline Mycorrhiza (R. irregularis) & $34.5^{\mathrm{cd}}$ & $38.2^{a b}$ & $1.15 \mathrm{f} \mathrm{g}^{\mathrm{g}}$ & $1.98^{b}$ & 3.29 ef & $4.56^{\mathrm{ab}}$ & 398 ef & $492^{a b}$ \\
\hline Mycorrhiza (G. fasciculatum) & $29.3^{\mathrm{f}-\mathrm{i}}$ & $34.8^{\mathrm{cd}}$ & $1.04 \mathrm{ghi}$ & 1.53 de & $2.82 \mathrm{fgh}$ & $3.90 \mathrm{~cd}$ & $365 \mathrm{fg}$ & $450^{\mathrm{cd}}$ \\
\hline Fish manure soil & $26.7^{\mathrm{ijk}}$ & $24.3^{\mathrm{klm}}$ & $0.76^{\mathrm{klm}}$ & $0.79 \mathrm{klm}$ & $1.68^{\mathrm{mn}}$ & $2.09^{j-m}$ & $251^{\mathrm{kl}}$ & $296^{\mathrm{ij}}$ \\
\hline Fish manure solution & $28.5^{g-j}$ & $26.5^{\mathrm{jkl}}$ & $0.79 \mathrm{klm}$ & $0.82^{\mathrm{i}-1}$ & $1.77 \mathrm{lmn}$ & $2.26^{\mathrm{ijk}}$ & $268^{\mathrm{jk}}$ & $318^{\text {hi }}$ \\
\hline Cattle manure soil & 27.7 hij & $28.2^{g-j}$ & $0.81 \mathrm{jkl}$ & $1.02^{g-j}$ & $1.95^{\mathrm{klm}}$ & 2.62 ghi & 289 ij & 343 gh \\
\hline Cattle manure solution & $30.8^{\text {efg }}$ & $30.2^{\mathrm{e}-\mathrm{h}}$ & $0.88^{\mathrm{h}-\mathrm{k}}$ & $1.16^{\mathrm{fg}}$ & $2.22^{\mathrm{i}-1}$ & 2.89 fgh & $307^{\mathrm{i}}$ & $371 \mathrm{f} \mathrm{g}^{\mathrm{g}}$ \\
\hline Vermicompost soil & $32.5^{\mathrm{de}}$ & $31.3^{\text {ef }}$ & $0.95^{\mathrm{g}-\mathrm{k}}$ & $1.66^{\mathrm{cd}}$ & 2.47 hij & 3.25 ef & $322 \mathrm{hi}$ & 396 ef \\
\hline Vermicompost solution & $37.5^{\mathrm{b}}$ & $40.5^{\mathrm{a}}$ & $1.59^{\mathrm{cd}}$ & $2.25^{\mathrm{a}}$ & $3.51^{\text {de }}$ & $4.85^{\mathrm{a}}$ & $417^{\text {de }}$ & $515^{a}$ \\
\hline Biofertilizer Phosphate & $30.5^{\text {efg }}$ & $32.5^{\mathrm{de}}$ & $1.31 \mathrm{ef}$ & $1.97^{\mathrm{b}}$ & 2.64 ghi & 3.58 de & $343 \mathrm{gh}$ & 420 de \\
\hline $\mathrm{LSD}_{5 \%}$ & \multicolumn{2}{|c|}{2.64} & \multicolumn{2}{|c|}{0.230} & \multicolumn{2}{|c|}{0.487} & \multicolumn{2}{|c|}{34.5} \\
\hline
\end{tabular}

Means with the same letters or means with differences less than LSD do not have statistically significant differences at the $5 \%$ level of probability. 


\subsection{Lateral Branch Number}

It was revealed by the comparison of the means that the highest number of lateral branches was related to the foliar application of vermicompost (29.58 branches), significantly differing from that of its soil application (20.91 branches) (Table 5). There were also significant differences between the mycorrhizal fungi so that the plants inoculated with $R$. irregularis produced $9 \%$ more lateral branches than those inoculated with G. fasiclutum (24.3 vs. 21.75 branches) (Table 5). However, the differences between the foliar and soil application of fish manure and cattle manure were not statistically significant (Table 5). Phosphate biofertilizer (19.25 branches) was classified in the same statistical group as the soil application of vermicompost (20.91 branches), which all differed from the control (11.8 branches) significantly (Table 5). A significant interaction was observed between year and species so that S. khuzestanica in the third year (26.5 branches) produced 59\% more lateral branches in the third year than S. rechingeri in the same year (16.6 branches) (Table 6).

\subsection{Leaf Area Index}

The highest leaf area index was 0.79 , obtained from the savory plants sprayed with vermicompost, but this treatment had no significant difference with the inoculation with $R$. irregularis, whose leaf area index was 0.76 . However, its difference from the treatment of vermicompost soil application (0.61) was significant (Table 5). The difference between the foliar and soil application of fish manure and cattle manure was insignificant (Table 5). Phosphate biofertilizer (0.63) was in the same statistical group as the soil application of vermicompost and inoculation with G. fasciculatum (0.67), but they all differed from the control (0.31) significantly (Table 5).

\subsection{Plant Dry Mass Weight}

Based on Table 5, the highest plant dry mass weight (including leaves and flowering branches) was $4.15 \mathrm{t} / \mathrm{ha}$, obtained from the foliar application of vermicompost, exhibiting an insignificant difference from the inoculation with $R$. irregularis ( $3.83 \mathrm{t} / \mathrm{ha})$ but a significant difference from the soil application of vermicompost (2.94 $t / h a)$. The foliar application of fish and cattle manure had no significant difference from their soil application (Table 5). Phosphate biofertilizer $(2.89 \mathrm{t} / \mathrm{ha}$ ) was ranked in the same statistical group as the soil application of vermicompost and inoculation with G. fasciculatum (3.36 t/ha), which all differed from the control (1.51 t/ha) significantly (Table 5$)$. There was a significant interaction between year and species so that $S$. khuzestanica in the third year had a $60 \%$ higher plant dry weight ( $3.7 \mathrm{t} / \mathrm{ha}$ ) than $S$. rechingeri in the same year with a plant dry weight of $2.3 \mathrm{t} /$ ha (Table 6).

\subsection{Essential Oil Percentage}

The comparison of the means showed that the foliar application of vermicompost was related to the highest essential oil percentage (5.53\%), which was in the same statistical group as inoculation with $R$. irregularis (5.25\%). However, it was not different from inoculation with other mycorrhizal fungi (Table 5). Phosphate biofertilizer $(4.43 \%)$ was placed in the same statistical group as inoculation with G. fasciculatum (4.78\%), and all differed from the control (2.45\%) significantly (Table 5). The foliar application of fish manure and cattle manure and their soil application did not exhibit any significant differences (Table 5). There was a significant interaction between year and species. The essential oil percentage of $S$ rechingeri in the third year $(5.1 \%)$ was $18 \%$ higher than that of this species in the second year $(4.3 \%)$ and $41 \%$ higher than that of S. khuzestanica in the third year (3.6\%) (Table 6).

\subsection{Carvacrol Content}

It was revealed that the savory plants sprayed with vermicompost had the highest carvacrol content $(85.54 \%)$, differing from the inoculation with $R$. irregularis insignificantly $(83.97 \%)$ and the soil application of vermicompost significantly $(80.26 \%)$ (Table 6). No significant difference was observed between the foliar application and soil application of cattle and fish manure (Table 5). The application of phosphate biofertilizer $(78.85 \%)$ and 
the foliar application of cattle manure $(78.19 \%)$ were statistically ranked in the same group, but they both differed from the control (72.08\%) significantly (Table 5). S. khuzestanica had $9 \%$ higher carvacrol content than S. rechingeri ( $82.62 \%$ vs. $75.5 \%)$ (Table 5$)$.

\subsection{Essential Oil Yield}

According to the comparison of the means, the highest essential oil yield was $22.92 \mathrm{~kg} / \mathrm{ha}$, obtained from the treatment of dissolved vermicompost, and the lowest was $3.74 \mathrm{~kg} / \mathrm{ha}$, obtained from the control (Table 5). The plants inoculated with $R$. irregularis had a $7 \%$ higher essential oil yield than those inoculated with F. mosseae and $40 \%$ higher than those inoculated with G. fasciculatum ( $20.09 \mathrm{~kg} / \mathrm{ha}$ vs. 18.77 and $16.10 \mathrm{~kg} / \mathrm{ha}$, respectively) (Table 5). There was not a significant difference between the application of phosphate biofertilizer and the soil application of vermicompost. There were no significant differences between the foliar application and soil application of cattle manure and fish manure (Table 5). The essential oil yield of $S$. rechingeri $(13.05 \mathrm{~kg} / \mathrm{ha})$ was $3 \%$ higher than that of $S$. khuzestanica $(12.65 \mathrm{~kg} / \mathrm{ha})$ (Table 5).

\subsection{Nitrogen Content}

The comparison of the means showed that the foliar application of vermicompost was related to the highest leaf $\mathrm{N}$ content $(3.78 \%)$, which was placed in the same statistical group as the inoculation with $R$. irregularis (3.47\%) but differed from the soil application of vermicompost significantly ( $2.58 \%$ ) (Table 5$)$. Phosphate biofertilizer $(2.63 \%)$ was not significantly different from the inoculation with G. fasciculatum (2.86\%), but both differed from the control (1.08\%) significantly (Table 5). The foliar and soil application of cattle manure and fish manure did not exhibit any significant differences (Table 5). A significant interaction was observed between year and species so that the leaf $\mathrm{N}$ content of S. khuzestanica in the third year $(2.7 \%)$ was $17 \%$ higher than that of S. khuzestanica in the second year $(2.3 \%)$ and $58 \%$ higher than (S. rechingeri) in the second year (1.7\%) (Table 6).

\subsection{Phosphorus Content}

The results disclosed that the highest leaf P content, 1.91\%, was related to the foliar application of vermicompost, which differed from the soil application of vermicompost $(1.35 \%)$ significantly (Table 5$)$. However, there was a non-significant difference between the foliar application and soil application of cattle manure and fish manure (Table 5). The phosphate biofertilizer (1.63\%) was ranked in the same statistical group as inoculation with $R$. irregularis $(1.56 \%)$, and both showed a significant difference from the control $(0.61 \%)$ (Table 5). Additionally, the results as to the interaction between year and treatment revealed that the plants sprayed with vermicompost in the third year had a $41 \%$ higher P content than those sprayed with vermicompost in the second year (2.25 vs. 1.59) (Table 7).

\subsection{Potassium Content}

As the comparison of the means revealed, the highest leaf $\mathrm{K}$ content of $4.18 \%$ was related to the foliar application of vermicompost, which was in the same statistical group as the inoculation with $R$. irregularis $(3.37 \%)$ but differed from the soil application of vermicompost (2.94\%) significantly (Table 5). The application of phosphate biofertilizer $(3.12 \%)$ had no significant difference from the inoculation with G. fasciculatum (3.37\%), but they both differed from the control $(1.43 \%)$ significantly (Table 5$)$. The differences between the foliar application and soil application of cattle manure and fish manure were insignificant (Table 5). Year and species interacted significantly so that the K content of S. khuzestanica in the third year (3.4\%) was $9 \%$ higher than its K content in the second year $(3.1 \%)$ and $70 \%$ higher than the K content of $S$. rechingeri in the second year ( $2 \%$ ) (Table 6$)$. Comparing the means for the interaction of year and treatment (Table 7) also indicated that the foliar application of vermicompost in the third year exhibited a $28 \%$ higher K content than its foliar application in the second year (4.85\% vs. $3.51 \%$ ). The control did not show any significant changes in its leaf K content between the second and third years (Table 7). 


\subsection{Iron Content}

As per the comparison of the means, the plants sprayed with vermicompost exhibited the highest leaf $\mathrm{Fe}$ content of $466.25 \mathrm{mg} / \mathrm{kg}$, and their difference from the plants inoculated with $R$. irregularis $(44.83 \mathrm{mg} / \mathrm{kg}$ ) was insignificant. However, they differed from the plants exposed to the soil application of vermicompost $(363.42 \mathrm{mg} / \mathrm{kg})$ significantly (Table 5). The application manner of cattle manure and fish manure brought about no changes in the leaf Fe content, as their foliar application and soil application did not differ significantly (Table 5). The phosphate biofertilizer $(381.5 \mathrm{mg} / \mathrm{kg})$ and the soil application of vermicompost were placed in the same statistical group, and both differed from the control (227.92 mg kg $\mathrm{k}^{-1}$ ) significantly (Table 5). The interaction between year and species was significant. S. khuzestanica in the third year with a leaf Fe content of $40.5 \mathrm{mg} / \mathrm{kg}$ outperformed this species in the second year $(348 \mathrm{mg} / \mathrm{kg}$ ) by $16 \%$, and S. rechingeri in the third year $(378 \mathrm{mg} / \mathrm{kg}$ ) by $7 \%$ (Table 6 ). The interaction between year and treat was significant, as the leaf Fe content increased by $23 \%$ in the plants sprayed with vermicompost in the third year $(515 \mathrm{mg} / \mathrm{kg})$, compared to the plants sprayed with the same fertilizer in the second year $(417 \mathrm{mg} / \mathrm{kg}$ ) (Table 7). The control plants did not differ in their Fe content between the first and second years (Table 7).

\section{Discussion}

The analysis of a random sample at the flowering stage detected 25 compounds in the essential oil of $S$. khuzestanica. The main compounds were carvacrol, $\beta$-myrcene, $\alpha$-terpinolene, $\gamma$-terpinene, linalool, thymol acetate, caryophyllene, $\beta$-bisabolene, and para-cymene, but carvacrol was the dominant compound, constituting $86.08 \%$ of the essential oil (Table 8). This is in agreement with the results of Sefidkon et al. [3] and Nooshkam et al. [16], who reported that the essential oil of this species contained $89 \%$ and $96 \%$ carvacrol, respectively. Similarly, Pellegrini et al. [31] reported that carvacrol, $\gamma$-terpinene, and para-cymene were the main constituents of savory. According to Table 8, 24 compounds were detected in S. rechingeri, but carvacrol was again the most abundant compound (76\%).

Ghasemi Pirbalout et al. [32] found that carvacrol (94.8\%) was the main compound in the essential oils of all the studied populations. Carvacrol has several biological effects: disinfectant, anti-inflammatory, sedative, antibacterial, antifungal, fermentation, and antioxidant activities [3]. We observed that all treatments increased the carvacrol content, but they did not influence other essential oil constituents. S. khuzestanica outperformed $S$. rechingeri in all traits, except for essential oil content and yield. The higher essential oil content of $S$. rechingeri can be ascribed to genetic differences because the ecological and soil conditions were similar for both species. The essential oil yield of the studied savory species was a function of the essential oil percentage and the leaf and flowering branch yield as the essential oil-containing part. So, any increase in these parameters can increase the essential oil yield. The results showed that both species responded to the treatments positively.

It seems that the effect of vermicompost on savory growth is related to its influence on increasing biological activities in the root zone, which affects the synthesis of growth regulators and improves $\mathrm{N}$ uptake. Heidarpour et al. [33] reported that the application of $2 t /$ ha vermicompost improved the morphophysiological parameters of summer savory. According to Neiji and Souri [21], the tallest savory plants resulted from 20\% vermicompost. In addition, the highest savory fresh weight was obtained from the application of vermicompost, but its dry weight was not affected. In another study, the highest plant height of summer-sown savory was obtained from the treatment of vermicompost [22]. In a similar study, Hossini et al. [20] reported that $S$. sahendica produced the highest essential oil percentage in $2 \mathrm{t} /$ ha vermicompost treatment. Improvement in the quality of essential fennel oil was reported by Osoli and Talesh [33], following the application of vermicompost. Boluin et al. [34] also found that vermicompost increased plant growth. There was a significant difference between the soil and foliar application of vermicompost. The foliar 
application increased the productivity of nutrients and tackles nutrient deficiencies faster than soil application.

Table 8. Essential oil compounds two species of savory.

\begin{tabular}{|c|c|c|c|c|c|}
\hline \multicolumn{3}{|c|}{ S. khusestanica } & \multicolumn{3}{|c|}{ S. rechingeri } \\
\hline Name & $\%$ & $\mathbf{R} \mathbf{I}$ & Name & $\%$ & R I \\
\hline$\alpha$-phellandrene & 0.57 & 539 & a-thujene & 0.2 & 924 \\
\hline$\alpha$-pipene & 0.26 & 560 & a-pinene & 0.2 & 936 \\
\hline$\beta$-myrcene & 1.17 & 680 & camphene & 0.2 & 946 \\
\hline$\alpha$-phellandrene & 0.16 & 735 & b-pinene & 0.4 & 974 \\
\hline$\alpha$-terpinolene & 0.59 & 761 & myrcene & 0.2 & 988 \\
\hline p-cymene & 2.03 & 787 & a-phellandrene & 0.3 & 1004 \\
\hline i-limonene & 0.15 & 795 & p-cymene & 3 & 1024 \\
\hline$\beta$-phellandrene & 0.12 & 804 & limonene & 0.3 & 1028 \\
\hline g-terpinene & 2.58 & 874 & 1,8-cineole & 2 & 1030 \\
\hline Trans-sabinene hydrate & 0.35 & 917 & g-terpinene & 5.8 & 1058 \\
\hline Linalool & 0.62 & 989 & cis-sabinene hydrate & 0.3 & 1065 \\
\hline Terpinene-4-01 & 0.49 & 1242 & terpinolene & 0.2 & 1086 \\
\hline$\beta$-terpineol & 0.12 & 1287 & trans-sabinene hydrate & 0.2 & 1097 \\
\hline$\alpha$-terpineol & 0.14 & 1304 & Linalool & 0.9 & 1098 \\
\hline Thymol methyl ether & 0.13 & 1393 & cumin aldehyde & 0.9 & 1143 \\
\hline Carvacrol & 86.08 & 1623 & borneol & 0.8 & 1165 \\
\hline$\alpha$-terpineol & 0.21 & 1707 & Terpinene-4-01 & 2.5 & 1174 \\
\hline Thymol acetate & 0.65 & 1759 & $\alpha$-terpineol & 0.8 & 1186 \\
\hline Linalyl acetate & 0.12 & 1779 & thymol methyl ether & 0.3 & 1232 \\
\hline Caryophyllene & 0.79 & 1911 & carvacrol methyl ether & 0.3 & 1242 \\
\hline Farnesene & 0.18 & 2107 & thymol & 0.2 & 1289 \\
\hline$\beta$-bisabolene & 1.86 & 2127 & carvacrol & 76 & 1298 \\
\hline$\alpha$-humulene & 0.31 & 2208 & $\beta$-caryophyllene & 0.5 & 1417 \\
\hline Caryophyllene oxide & 0.10 & 2351 & $\beta$-bisabolene & 0.4 & 1505 \\
\hline i-t-butyl-4-benzene & 0.21 & 3407 & - & & \\
\hline
\end{tabular}

Among the mycorrhizal species, $R$. irregularis was significantly superior over the other species. It can be said that different plant species respond to various mycorrhizal fungi differently, so research should instead use local fungi that are symbiotic to the plants native to a similar location. In a study, the highest plant dry mass weight of S. khuzestanica was observed in pots containing the mycorrhizal fungi [18]. In another study, it was revealed that mycorrhiza increased the forage production of Artemisia [35]. Nonetheless, Nooshkam et al. [16] reported that mycorrhizal inoculation had no effects on increasing the plant height and lateral branches of $S$. khuzestanica. In the present study, inoculation with mycorrhiza improved the leaf N, P, K, and Fe contents of both savory species. Gupta et al. [17] reported that mycorrhiza increased N, P, and K uptake by mint plants. In another study, the results showed that mycorrhiza increased the $\mathrm{P}$ and Fe content of Artemisia [36]. It was also reported that mycorrhiza increased the $\mathrm{P}$ content of corn, and F. mosseae was the most effective among the species [36]. Zamani et al. [37] indicated that the application of biofertilizers increased the essential oil content of fennels. However, Nooshkam et al. [16], who studied S. khuzestanica, Karayiannis et al. [38], who studied Origanum majorana, and Andrea [39], who studied Ocimum basilicum, reported that inoculation with mycorrhiza had no significant impact on essential oil content and composition. Various mechanisms were mentioned for the effect of mycorrhiza on the vegetative growth of plants, for instance, its impact on the uptake of nutrients, e.g., N, P, and K, from soil [40]. Mycorrhiza symbiosis with the roots increases photosynthesis by enhancing the uptake of water and nutrients, contributing to the synthesis of more assimilates and growth improvement [41].

Based on the results, the application of phosphate biofertilizer outperformed cattle and fish manure. The superiority of phosphate biofertilizer over manure was also proven by Neiji and Souri [21] in a study on savory. In agreement, Saleh [10] stated that phosphate 
biofertilizer can increase the solubility of phosphorous compounds precipitated in soil by specific processes, and this phosphorus meets a part of the plant requirement. On the other hand, this fertilizer contains beneficial phosphate-solubilizing bacteria that acidify the soil and exudate phosphatase, thereby releasing phosphate ions from phosphorus-containing compounds, which are absorbable by plants. He stated that, in addition to increasing fertilizer uptake efficiency, phosphate biofertilizer significantly increases yield, too. The application of cattle and fish manure was effective versus the control in which no fertilizer was applied. The foliar and soil application of fish and cattle manure did not cause any significant differences in any trait, but their soil application was more effective in the third year than in the second year. The incorporation of fish fertilizer into the soil as an organic fertilizer positively affects the physical and chemical characteristics of soil. This is of high importance, especially regarding the increased water availability following an increase in soil porosity [18]. A similar investigation [42,43] recommends animal and fish manure for soil amendment. The data of the first year were not discussed here, due to plant establishment. Significant increases occurred for all traits in the third year versus the second year, which may be associated with the re-application of fertilizers in the second and third years or the gradual release of nutrients from biological and organic fertilizers, which improved fertilization efficiency.

\section{Conclusions}

Based on the results, the studied savory species differed in the main and percentages of the essential oil constituents. The essential oil yield of these savory species is a function of the essential oil percentage and the leaf and flowering branch yield as the essential oil-containing part; so any increase in these components can increase the essential oil yield. The foliar application of vermicompost provided the best conditions for the growth and development, essential oil percentage, and nutrient contents of two savory species. The inoculation with mycorrhiza and phosphate biofertilizer provided the second and third best conditions, respectively. According to the results, both S. khuestanica and rechingeri cultivars were high in dry matter yield and essential oil percentage. Therefore, depending on the purpose of cultivation, it can be recommended as to which species should be used in this area.

The application of biological and organic fertilizers tends to partially meet the food requirement of this invaluable native medicinal herb and contribute to the better establishment of soil-borne microorganisms for subsequent rotations with minimum environmental damages, while helping the sustainability and health of the agricultural system.

Author Contributions: Conceptualization, supervision, and administration, R.A.; methodology and formal analysis, A.B.; writing, original draft, R.A. and A.B.; writing, editing, R.Z.S. and H.A.E.E. All authors have read and agreed to the published version of the manuscript.

Funding: H.A.E would like to thank RMC, Universiti Teknologi Malaysia (UTM), Allcosmos Industries Sdn. Bhd. and Arif Efektiv Sdn. Bhd. with project No. R.J130000.7344.4B200 and R.J130000.7609.4C187.

Institutional Review Board Statement: Not applicable.

Informed Consent Statement: Not applicable.

Data Availability Statement: Not applicable.

Conflicts of Interest: The authors declare no conflict of interest.

\section{References}

1. Hadian, J.; Azizi, A.; Tabatabaei, M.F.; Naghavi, M.R.; Jamzad, Z.; Friedt, W. Analysis of the genetic diversity and affinities of different Iranian Satureja Species based on SAMPL markers. J. Med. Plant Res. 2010, 76, 1927-1933. [CrossRef]

2. Jamzad, Z. Thymus and Satureja Species of Iran; Publications of Research Institute of Forests and Rangelands: Tehran, Iran, 2009; 171p. 
3. Sefidkon, F.; Sadeghzadeh, L.; Timoori, M.; Asgari, F.; Ahmadi, S. Evaluation the effects of antimicrobial of essential oil of two savory species Satureja bachtiarica Bunge and Satureja khuzistanica Jamzad in two harvest stage. J. Med. Aromat. Plants 2007, 23, 174-182.

4. Tavafi, M.; Ahmadvand, H.; Tamjidipoor, A.; Delfan, B.; Khalatbari, A.R. Satureja khozestanica essential oil ameliorates progression of diabetic nephropathy in uninephrectomized diabetic rats. Tissue Cell 2011, 43, 45-51. [CrossRef] [PubMed]

5. Samadi, N.; Zaree, R.; Bakhiar, H.; Salehnia, A.; Azimi, S. Comparative Antibacterial Efficacy of Endemic Satureja Khuzistanica Jamzad Essential Oil, Sodium Hypochlorite and Chlorhexidine Gluconate Solutions as Root Canal Irrigations. Dent. Res. J. 2011, $8,28-32$.

6. Sepahvand, A.; Kordbache, P.; Delfhan, B.; Zeini, F.; Hashemi, S.; Mahmoodi, M. Antifungal effects of essential oil of Satureja khuzistanicais in the region of Lorestan with the method of in vitro. Iran. J. Yafteh. Lorestan Univ. Med. Sci. 2005, 2, 37-43.

7. Fecka, I.; Turek, S. Determination of polyphenolic compounds in commercial herbal drugs and spices from Lamiaceae: Thyme, wild thyme and sweet marjoram by chromatographic techniques. Food Chem. 2008, 108, 1039-1053. [CrossRef]

8. Glauce, M.; Sokmen, M.; Daferera, D.; Agar, G.; Ozkan, H.; Kartal, N. In vitro antibacterial, antifungal, and antioxidant activities of the essential oil and methanol extracts of herbal parts and callus cultures of Satureja hortensis L. J. Agric. Food Chem. 2003, 51, 3958-3965. [CrossRef] [PubMed]

9. Eminagaoglu, O.; Tepe, B.; Yumrutas, O.; Akpulat, H.A.; Daferera, D.; Polissiou, M. The in vitro antioxidative properties of the essential oils and methanol extracts of Satureja spicigera (K. Koch.) Boiss. and Satureja cuneifolia ten. Food Chem. 2007, 100, 339-343. [CrossRef]

10. Saleh Rastin, N. Biofertilizers and Their Role in Order to Reach to Sustainable Agriculture. In A Compilation of Papers of Necessity for the Production of Biofertilizers in Iran; Klavazi, K., Malakouti, J., Eds.; Nashr, Amouzesh Keshavarzi: Tehran, Iran, 2001; 54p.

11. Heidari, Z.; Besharati, H.; Farahani, S.M. Effect of some chemical fertilizer and biofertilizer on quantitative and qualitative characteristics of saffron. Saffron Agron. Technol. 2014, 2, 187-189. [CrossRef]

12. Liu, R.; Lal, R. Potentials of engineered nanoparticles as fertilizers for increasing agronomic productions. Sci. Total Environ. 2015 514, 131-139. [CrossRef]

13. Gupta, M.; Kiran, S.; Gulati, A.; Singh, B.; Tewari, R. Isolation and identification of phosphate solubilizing bacteria able to enhance the growth and aloin-a biosynthesis of Aloe barbadensis Miller. Microbiol. Res. 2012, 167, 358-363. [CrossRef]

14. Perramon, B.; Bosch-Serra, A.D.; Domingo, F.; Boixadera, J. Organic and mineral fertilization management improvements to a double annual cropping system under humid Mediterranean conditions. Eur. J Agron. 2016, 76, 28-40. [CrossRef]

15. Zaidi, A.; Saghir Khan, M.D.; Amil, M.D. Interactive effect of diazotrophic microorganisms on yield and nutrient uptake of chickpea (Cicer arietinum L.). Eur. J. Agron. 2003, 19, 15-21. [CrossRef]

16. Nooshkam, A.; Majnonhossini, N.; Hadian, j.; Jahansoz, M.R.; Khavazi, K.; Salehnia, A.N.; Hedayatpoor, S. Study the effects of biological and chemical fertilizer on quantitative and qualitative characteristics of savory species (Satureja khuzestanica jamzad). J. Crop Prod. 2016, 84, 87-103.

17. Gupta, M.L.; Prasad, A.; Ram, M.; Kumar, S. Effect of the vesicular-arbuscular mycorrhizal (VAM) fungus Glomus fasciculatum on the essential oil yield-related characters and nutrient acquisition in the crops of different cultivars of menthol mint (Menthe arvensis) under field conditions. Bioresour. Technol. 2004, 81, 77-79. [CrossRef]

18. Ahmadabadi, Z.; Ghajarsepanloo, M. Effect of organic fertilizer application on some soil physical properties. J. Soil Water Conserv. Res. 2012, 12, 99-116.

19. Heidarpour, O.; Esmaielpour, B.; Soltani Toolarood, A.; Khorramdel, S. Effect of vermicompost on summer's morphophysiological, biochemical, and yield characteristics savory (Satureja hortensis L.) under different irrigation regimes. J. Agroecol. 2020, 12, 507-522. [CrossRef]

20. Hossaini, S.M.; Aghaalikhani, M.; Sefidkon, F.; Galivant, A. Vegetative and essential oil yields of savory (Satureja sahendica Burn.) affected by vermicompost and Redroot Pigweed (Amaranthus retroflexux L.) competition. Iran. J. Med. Aromat. Plants Res. 2015, 31, 342-356. [CrossRef]

21. Naiji, M.; Souri, M.K. Evaluation of growth and yield of Savory (Satureja hortensis) under organic and biological fertilizers toward organic production. J. Plant Prot. 2015, 38, 93-103. [CrossRef]

22. Rezvani Moghadam, P.; Amin Ghafoori, A.; Bakhshaei, S.; Jafari, L. Study the effects of biological and organic fertilizers on quantitative traits and essential oil of savoy (Satureja hortensis L.). J. Agric. 2013, 5, 105-112. (In Persian) [CrossRef]

23. Tohidi Nejad, E.; Rastegari, F. Effects of biological and organic fertilizers on morphological parameters and chamazulene yield of German chamomile (Matricaria chamomilla L.) under drought stress condition. Iran. J. Med. Aromat. Plants 2019, 34, 949-962. [CrossRef]

24. Miri, H.; Darzi, M.T. Effects of manure and phosphate solubilizing biofertilizer on growth, yield and essential oil quality of dragonhead (Dracocephalum moldavica L.) in Firouzkuh region. Field Crops Res. 2017, 49, 35-45. [CrossRef]

25. Arvanitoyannis, I.S.; Kassaveti, A. Fish industry waste: Treatments environmental impacts, current and potential uses. Int. J. Food Sci. Technol. 2008, 43, 726-745. [CrossRef]

26. El-Tarabily, A.K.; Nassar, A.H.; Giles, E.; Hardy, S.J.; Sivasithamparam, K. Fish emulsion as a food base for rhizobacteria promoting the growth of radish (Raphanus sativus L. var. sativus) in a sandy soil. Plant Soil 2003, 252, 397-411. [CrossRef]

27. Aranganathan, L.; Radhika Rajasree, S.R. Bioconversion of marine trash fish (MTF) to organic liquid fertilizer for effective solid waste management and its efficacy on Tomato growth, Manag. Environ. 2016, 27, 93-103. [CrossRef] 
28. Shahsavani, S.; Abbaspoor, A.; Parsaiyan, M.; Younkers, Z. Effect of fish waste, chemical fertilizer and biofertilizer on yield and yield components of bean (Vigna sinensis) and some soil properties. J. Pulses Res. 2017, 8, 45-59. (In Persian) [CrossRef]

29. Sparkman, O.D. Identification of essential oil components by gas chromatography/quadrupole mass spectroscopy Robert P. Adams. J. Am. Soc. Mass Spectrom. 2005, 16, 1902-1903. [CrossRef]

30. Emami, A. Methods of Plant Analysis (Volume I). J. Inst. Soil Water 1996, 2, 128. (In Persian)

31. Pellegrini, M.; Ricci, A.; Serio, A.; Chaves-López, C.; Mazzarrino, G.; D’Amato, S.; Paparella, A. Characterization of essential oils obtained from abruzzo autochthonous plants. Antioxidant and antimicrobial activities assessment for food application. Foods 2018, 7, 19. [CrossRef]

32. Ghasemi Pirbalouti, A.; Moalem, E.; Yousefi, M.; Malekpoor, F.; Yousef-Naanaie, S. Influence of 315 ecological factors on carvacrol content of Satureja khuzistanica Jamzad. J. Essent. Oil Bear. Plants 2014, 14, 630-638. [CrossRef]

33. Osoli, N.; Taleshi, K. Evaluation of the effects of biological fertilizers and vermicompost on biological characteristics and essential oil quality of fennel plant (Foeniculum vulgare Mill) Khoramabad. J. Appl. Res. Plant Ecophysiol. 2018, 4, 123-138. (In Persian)

34. Blouin, M.; Barrere, J.; Meyer, N.; Lartigue, S.; Barot, S.; Mathieu, J. Vermicompost significantly affects plant growth. A metaanalysis. J. Sustain. Agric 2019, 39, 34. [CrossRef]

35. Chaudhary, V.; Kapoor, R.; Bhatnagar, A.K. Effectiveness of two arbuscular mycorrhizal fungi on essential oil and artemisinin concentrations in three accessions of Artemisia annua L. Appl. Soil Ecol. 2008, 40, 174-181. [CrossRef]

36. Amiri, P.; Azizi, M.; Nabizade, A. Effect of mycorrhizal fungi on yield and quality of maize under drought stress conditions. J. Agric. Sci. 2012, 91, 32-48.

37. Zamani, F.; Amirnia, R.; Rezaei-Cheyanne, E.; Rahimi, A. The effect of bacterial biofertilizers and mycorrhizal fungi on seed yield and chemical composition of essential oil of three fennel landrace. J. Crops Improv. 2019, 20, 831-848. [CrossRef]

38. Karagiannidisa, N.; Thomidisa, T.; Lazarib, D.; Panou-Filotheoua, E.; Karagiannidoua, C. Effect of three Greek arbuscular mycorrhizal fungi in improving the growth, nutrient concentration, and production of essential oils of oregano and mint plants. Sci. Hort. 2011, 129, 329-334. [CrossRef]

39. Andrea, C.; Lingua, G.; Bardi, L.; Masoero, G.; Berta, G. Influence of arbuscular mycorrhizal fungi on growth and essential oil composition in Ocimum basilicum var. Genovese. Caryologya 2007, 60, 106-110. [CrossRef]

40. Abdelhafez, A.; Abdel-Monsief, R.A. Effects of VAmycorrhizal inoculation on growth, yield and nutrient content of cantaloupe and cucumber under different water regimes. J. Agric. Biol. Sci. 2006, 2, 503-550.8.

41. Khalvati, M.A.; Mzafar, A.; Schmidhalter, U. Quantification of water uptake by arbuscular mycorrhizal hypha and its signification for leaf growth, water relations, and gas exchange of barley subjected to drought stress. Plant Biol. 2005, 706-712. [CrossRef]

42. Xu, Y.; Ding, F.; Gao, X.; Wang, Y.; Li, M.; Wang, J. Mineralization of plant residues and native soil carbon as affected by soil fertility and residue type. J. Soils Sediments 2019, 19, 1407-1415. [CrossRef]

43. Safikhani, F.; Heydari sharifabad, H.; Syadat, A.; Sharifi ashorabadi, A.; Syednedjad, M.; Abbaszadeh, B. The effect of drought stress on percentage and yield of essential oil and physiological characteristics (Deracocephalum moldavica L.). J. Med. Aromat. Plant 2007, 23, 86-89. 